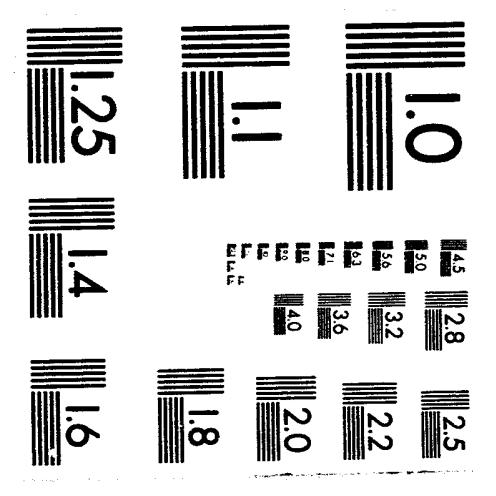



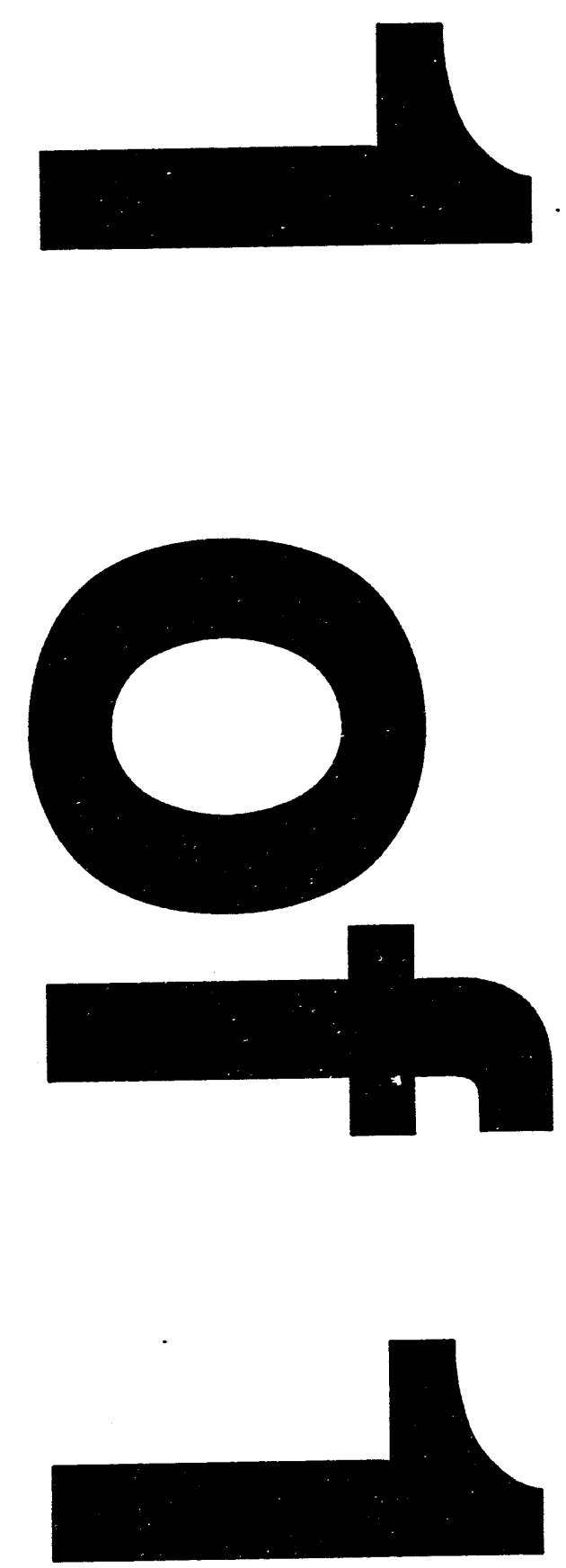


\title{
AN IMPROVED CBOICE OF OSCILLATOR BASIS FOR BANANA SHAPED NUCLIDES
}

\author{
R. R. CHASMAN
}

Physics Division. Argonne National Laboratory, Argonne, IL 50439-4843

(Received

\begin{abstract}
The question of the appropriate choice of oscillator basis functions for studying exotic nuclear shapes is raised. Difficulties with the conventional choice of oscillator basis states are noted for shapes having a large banana component. A prescription for an improved oscillator basis to study these shapes is given. It can be applied in a more general context. New calculations with this improved basis are presented for the banana deformation mode. The change of basis gives results that improve the prospects of finding states in the banana minimum for many isotopes of $\mathrm{Tl}, \mathrm{Pb}$ and $\mathrm{Bi}$.
\end{abstract}

Recently, we have found [1] that extending the deformation space used in cranked strutinsky calculations to include banana $\left(Y \frac{1}{3}(\theta, \phi)\right)$ deformations, gives new minima that are, in many cases, lower than the minima that have been predicted [2] and found [3] for high-spin axially symmetric superdeformed states in nuclides near $A=190$. Our motivation for the study of exotic deformation modes comes from the observation [4] of superdeformed bands in nuclides such as $198 \mathrm{~Pb}$. Our calculations suggest that they should be far above yrast at $I=40$, where the superdeformed bands are being populated. There have been predictions $[5,6,7]$ of axially symmetric superdeformed states at $I=0$ for these nuclides and the existence of such states at $I=0$ remains an interesting, open question. 
The banana minima are characterized by shapes with moderate values of the quadrupole deformation parameter and fairly large values of the banana deformation parameter. The states in this minimum were found to have large moments of inertia and very large quadrupole moments; 50\% larger than the quadrupole moments that were measured [3] for the high-spin axially symmetric superdeformed states of this mass region. Although these large quadrupole moments are surprising because the quadrupole term in the shape expansion is not large, we can calculate them in two independent ways: (1) microscopically, by summing single-particle quadrupole moments; (2) macroscopically, by calculating a quadrupole moment from the moments $\left\langle X_{1}^{2}\right\rangle$ of the liquid drop shape. Specifically, we found that at the banana minimum, the quadrupole term of the shape expansion was typically $\nu_{2}=0.35$, but the calculated quadrupole moment is the same as that of a pure quadrupole shape with $\nu_{2} \cong 0.8$. This large difference in quadrupole moments should make one wonder about the adequacy of a conventional choice of oscillator basis functions; i.e. oscillator basis functions having the same deformation as the quadrupole term in the shape expansion. We have explored this point and find that, for large banana deformations, the energies of single-particle states near the fermi level converge slowly with increasing shell number and one must diagonalize arrays that are $1500 \times 1500$ to get stable energy level differences using the conventional choice of an oscillator basis. This is impractical for detailed studies of energy surfaces. One might hope that a better choice of oscillator basis would allow the use of smaller matrices to get reliable results. As the study of nuclear energy surfaces is extended to larger and more exotic deformation spaces in the future, the need for a better choice of oscillator basis functions will become a pressing issue. This need is particularly great for $Y \frac{1}{3}(\theta, \phi)$ deformations with cranking about the $Y$-axis, which is the preferred 
axis for cranking. In this case, all symmetries are broken and the matrices to be diagonalized are not decomposable.

We denote the quadrupole deformation of the shape expansion as $\left(\nu_{2}\right)_{s}$ and that of the oscillator basis as $\left(\nu_{2}\right)_{b}$. The deformation parameters $\nu_{2}$ have been defined [8] previously. Rather than increasing the basis space as discussed above, we here investigate the choice of basis. The need to use a large basis for large $Y_{3}^{\frac{1}{3}}(\theta, \phi)$ deformations is analogous to the need to use a large spherical basis for the description of deformed quadrupole shapes. If, however, the deformation of the basis is chosen such that $\left(\nu_{2}\right)_{b}=\left(\nu_{2}\right)_{s}$, the size of the basis needed for a calculation involving purely quadrupole shapes is essentially independent of the deformation. Similarly by making systematic variations in $\left(\nu_{2}\right)_{b}$, for nuclei with large $Y \frac{1}{3}(\theta, \phi)$ deformations, we find that one can get large improvements in the calculated single particle spectrum. We find that a near optimum choice comes from equating the sum of the three moments $\left\langle x_{1}^{2}\right\rangle$ of the actual shape that we are studying, to those of an equivalent axially symmetric quadrupole shape. We then use this quadrupole shape to define the magnitude of the basis deformation; $i . e$. we set

$$
\left\langle R^{2}\right\rangle_{b}=\left\langle R^{2}\right\rangle_{s}
$$

where $\left\langle R^{2}\right\rangle_{s}$ denotes the sum of the three moments for the shape of interest. Using units such that $\left\langle X_{1}^{2}\right\rangle=1$ for a spherical shape, we note that for a purely quadrupole shape

$$
\left\langle x_{i}^{2}\right\rangle=\left(w_{i}\right)^{-2}
$$

with

$$
\left(w_{i}\right)^{-2}=\exp \left((4 / 3) \nu_{2} \cos \left(\gamma+(i-1) 120^{\circ}\right)\right)
$$


setting $i=1$ for siee $z$ direction; $i=2$ for the $X$ direction; and $i=3$ for the $Y$ direction. For the more general shapes of interest here, the three moments are obtained numericaity. From these equations, we determine only the quadrupole deformation of the basis, $\nu_{2}$. The quantity $\left\langle R^{2}\right\rangle$ is weakly dependent on $\gamma$, and we set $\gamma$ equal to zero in eq. (3), for purposes of approximating $\left(\nu_{2}\right)_{b}$. However, in the case of the $Y \frac{1}{3}(\theta, \phi)$ deformation, there is no cylindrical symmetry and we can further improve the basis with a choice of $\gamma \neq 0$. We determine an effective value of $\gamma$ for each of the three directions, using eqs. (2) and (3) with the now determined value of $\left(\nu_{2}\right)_{b}$, and the values of $<x_{1}^{2}>s$. There is an ambiguity in the sign of $\gamma$ as calculated from eq. (3) for the $z$ direction. We choose this sign to be positive for shapes with $\left.\left\langle Y^{2}\right\rangle\right\rangle\left\langle\mathrm{X}^{2}\right\rangle$ and negative for $\left.\left\langle x^{2}\right\rangle\right\rangle\left\langle y^{2}\right\rangle$. When the two are equal, we set $\gamma_{z}=0$. We then choose $(\gamma)$ for the basis as the average of the values that we get for the three directions. The procedure can be iterated for values of $\gamma_{b}$ near $60^{\circ}$, now using the value of $\gamma_{b}$, and redetermining $\left(\nu_{2}\right)_{b}$. This prescription gives a good choice for $\gamma$ for several cases in which we have examined the dependence of eigenvalues on the choice of $\boldsymbol{\gamma}$. We note that $\gamma$ is fairly large $\left(\sim-15^{\circ}\right)$ for $\nu_{31}=0.525$ and the advantages of using a cartesian oscillator basis are substantial. As we are using a cartesian oscillator basis, we could simply define three independent oscillator frequencies for the three directions, without recourse to a single value of $\gamma$. However, we find that for a given basis size, using the average value of $\gamma$, gives better energies than the use of three independent frequencies.

It is interesting to see if the insight that we get from banana shapes on $\left(\nu_{2}\right)_{b}$ applies for conventional shapes. We have carried out energy level calculations for a shape characterized by $\nu_{2}=1$. and $\nu_{4}=0.1$. The sum of the moments for this shape is the same as that of a pure quadrupole shape with 
$\nu_{2} \cong 0.75$. Using an extremely truncated basis set, we indeed Eind that the calculated eigenvalues are minimized for $\left(\nu_{2}\right)_{b} \cong 0.75$. However, using basis sets of the size normally used in Woods-Saxon calculations, the calculated energy levels are essentially the same for $\left(\nu_{2}\right)_{b}=1.0$ as for $\left(\nu_{2}\right)_{b}=0.75$.

Using our prescription for the oscillator basis, we have recalculated the shell corrections for $\nu_{31}$ values of $0.375,0.450$ and 0.525 . The details of the calculation have been described [1] previously. The largest effects show up for $\nu_{31}=0.525$, where the large negative shell corrections obtained previously are reduced considerably. We can understand this as arising from the fact that the conventional basis choice has a poor overlap with the eigenstates and the level density near the fermi level calculated with this basis is too low. Using our prescription increases $\left(\nu_{2}\right)_{b}$ by $\sim+0.6$ relative to the conventional choice for $\nu_{31}=0.525$. The changes in the shell corrections are moderate for $\nu_{31}=0.45$. The major effect is that the typical banana minimum is shifted from $\left(\nu_{2}\right)_{s}=0.35$ to $\left(\nu_{2}\right)_{s}=0.30$. For $\nu_{31}=0.375$, the changes in energy levels and shell corrections are fairly small. For yet smaller values of $\nu_{31}$, we note that the change in $\left(\nu_{2}\right)_{b}$ relative to the standard choice depends quadratically on $\nu_{31}$, and the mismatch between the shape and the basis choice does not give rise to significant effects for values of $\nu_{31}$ of 0.3 or lower for $\left(\nu_{2}\right)_{\mathrm{s}}$ values near the banana minimum.

This decrease in shell corrections for $\nu_{31}=0.525$ has the rather beneficial effect of shifting the banana minimum in many isotopes of $\mathrm{Pb}$ and $\mathrm{Bi}$ from $\nu_{31}=0.525$ to the value of $\nu_{31}=0.45$. The increase in total energy for the largest values of $\nu_{31}$ indicates that there is a barrier to fission going via this mode. With the shifts in the values of the deformation parameters at the banana minimum, the quadrupole moments and moments of inertia of bands in the banana minimum are now calculated to be somewhat smaller than previously in the 
lighter isotopes of $\mathrm{Tl}, \mathrm{Pb}$ and $\mathrm{Bi}$. For a typical deformation $\left(\nu_{2}=0.30\right.$, $\left.\nu_{31}=0.45\right)$, the calculated quadrupole moments are $30 z$ larger than those obtained for the axially symmetric superdeformed states in this mass region; rather than $\sim 50 z$ as found previously with the conventional choice of $\left(\nu_{2}\right)_{b}$.

In fig. I, we display the well depth of the banana minimum at $I=30$. This is the barrier between the banana minimum and axially symmetric shapes. We also show the vaiues of $\nu_{2}$ and $\nu_{31}$ at the banana minimum. Apart from the heavy $B i$ isotopes, the minimum in the energy occurs for values of $\nu_{31}$ less than 0.525 .

In fig. 2, we show the excitation of the banana minimum relative to the prolate axially symmetric minimum at $I=40$, which is typically the angular momentum brought into the compound nucleus in the heavy ion reactions used to study this region. Note that for most isotopes with neutron number $N \geq 115$, the banana minimum is quite excited relative to the prolate minimum and it is hard to envisage the population of such states. We also show in this figure static moments of inertia at $I=35$, calculated without pairing.

In figs. (3) and (4), we show single particle neutron and proton level schemes as a function of rotational frequency.

In fig. (5), we show the neutron alignments <Jy> for neutron numbers between 106 and 119; in fig. (6), we show the proton alignments for proton numbers between 77 and 84 . There are very few orbitals with alignments greater than 1, even at $I=50$. The changes in the single-particle alignments are rather small between $I=20$ and $I=35$, typically less than $0.1 \mathrm{~h}$.

In table 1, we give the properties of those nuclides that look most promising for experimental investigation. $\Delta \mathrm{E}_{\min }$ is the difference in energy between the axially symmetric superdeformed minimum and the banana minimum. A negative value means that the banana minimum is the lower of the two. We note 
that for the $\mathrm{Hg}$ isotopes the banana and axially symmetric superdeformed minima are rather close in energy for $N>110$, and might mix.

In searching for rotational bands associated with banana minima, it is important to note that both the pairing correlation energy and the moments of inertia are larger for configurations in the banana minimum tian for those having axially symetric superdeformed shapes. This means that the effects of pairing are more important in determining both the static and dynamic moments of inertia of banana shapes than for axially symmetric superdeformed shapes. We can estimate moments of inertia with pairing using the approach of ref. [9]. For this estimate, we have carried out a cranked strutinsky calculation about the $Y$-axis, for the deformation $\nu_{2}=0.30, \nu_{4}=-0.02$, and $\nu_{31}=0.45$. For ${ }^{192} \mathrm{Hg}$, this gives a static moment of $95 \mathrm{~h}^{2}(\mathrm{MeV})^{-1}$ at low spins, increasing to 120 at $I=40$. We get a dynamic moment of $\sim 100 \mathrm{~h}^{2}(\mathrm{MeV})^{-1}$ at $\mathrm{I}=10$, increasing to $\sim 140$ at $I=30$. Above $I=30$, the dynamic moment continues to increase attaining values of $\sim 160$ at $I=50$. As the alignments of individual orbitals are small, these estimates are relevant for neighboring nuclides having the same shape. A more quantitative estimate of the moments of inertia requires an extension of the variational space to include additional deformation modes and also requires that the cranking be carried out about the Y-axis for all shapes.

There is a need to investigate the extent to which the properties of bands in the banana minimum are modified by the inclusion of additional multipoles in the deformation space. This task will be expedited by utilizing the choice of oscillator basis given in this work.

It is a pleasure to thank I. Hamamoto and $S$. Landowne for helpful discussions on this problem. The calculations reported here were carried out on the NERSC Cray computer facility at Livermore. This research was supported by the U.S. Department of Energy, Nuclear Physics Division, under contract W$31-109-E N G-38$. 
[1] R. R. Chasman, Phys. Lett. B266 (1991) 243.

[2] R. R. Chasman, Phys. Lett. B219 (1989) 227.

[3] E. F. Moore et al., Phys. Rev. Lett. 63 (1989) 227.

[4] T. F. Wang et al., Phys. Rev. C 43 (1991) R2465.

[5] C. F. Tsang and S. G. Nilsson, Nucl. Phys, A140 (1970) 225.

[6] M. Girod, J. P. Delaroche and J. F. Berger, Phys. Rev. C 38 (1988) 1519.

[7] P. Bonche et al., Nucl. Phys. A500 (1989) 308.

[8] R. R. Chasman, I. Ahmad, A. M. Friedman and J. R. Erskine, Rev. Mod. Phys. 49 (1977) 833; see Appendix A.

[9] R. R. Chasman, Phys. Lett. B242 (1990) 317.

\section{DISCLAIMER}

This report was prepared as an account of work sponsored by an agency of the United States Government. Neither the United States Government nor any agency thereof, nor any of their employees, makes any warranty, express or implied, or assumes any legal liability or responsibility for the accuracy, completeness, or usefulness of any information, apparatus, product, or process disclosed, or represents that its use would not infringe privately owned rights. Reference herein to any specific commercial product, process, or service by trade name, trademark, manufacturer, or otherwise does not necessarily constitute or imply its endorsement, recommendation, or favoring by the United States Government or any agency thereof. The views and opinions of authors expressed herein do not necessarily state or reflect those of the United States Government or any agency thereof. 
Table I

\begin{tabular}{|c|c|c|c|c|c|c|c|}
\hline 2 & $\mathrm{~N}$ & $\nu_{2}$ & $\nu_{31}$ & $\begin{array}{l}\left(E^{*}\right) 40 \\
\mathrm{MeV}\end{array}$ & $\begin{array}{c}\text { (W.D. ) } 30 \\
\mathrm{MeV}\end{array}$ & $\begin{array}{c}\Delta \mathrm{E}_{\mathrm{min}} \\
\mathrm{MeV}\end{array}$ & $\begin{array}{c}(1)_{35} \\
h_{2}(\mathrm{MeV})^{-1}\end{array}$ \\
\hline $78 \mathrm{pt}$ & 112 & 0.30 & 0.450 & -0.9 & 1.3 & -0.9 & 133 \\
\hline \multirow[t]{4}{*}{${ }^{79} \mathrm{Au}$} & 110 & 0.30 & 0.450 & -1.6 & 1.7 & -1.4 & 135 \\
\hline & 111 & 0.30 & 0.450 & -1.4 & 1.5 & -0.9 & 138 \\
\hline & 112 & 0.30 & 0.450 & -1.0 & 1.6 & -0.7 & 138 \\
\hline & 113 & 0.30 & 0.450 & -0.5 & 1.5 & -0.8 & 141 \\
\hline \multirow[t]{7}{*}{$80 \mathrm{Hg}$} & 108 & 0.30 & 0.375 & -1.3 & 1.4 & -1.1 & 125 \\
\hline & 109 & 0.30 & 0.450 & -1.1 & 1.6 & -0.9 & 135 \\
\hline & 110 & 0.30 & 0.450 & -1.2 & 1.8 & -1.0 & 136 \\
\hline & 111 & 0.30 & 0.450 & -0.7 & 2.1 & -0.4 & 139 \\
\hline & 112 & 0.30 & 0.450 & -0.1 & 2.0 & -0.1 & 139 \\
\hline & 113 & 0.30 & 0.450 & 0.3 & 1.6 & -0.2 & 143 \\
\hline & 114 & 0.30 & 0.450 & 0.9 & 1.3 & 0.1 & 142 \\
\hline \multirow[t]{7}{*}{${ }^{81} \mathrm{Tl}$} & 108 & 0.30 & 0.375 & 0.0 & 1.9 & -1.9 & 125 \\
\hline & 109 & 0.30 & 0.450 & 0.2 & 1.8 & -1.7 & 135 \\
\hline & 110 & 0.30 & 0.450 & 0.1 & 2.3 & -1.8 & 137 \\
\hline & 111 & 0.30 & 0.450 & 0.5 & 2.2 & -1.5 & 139 \\
\hline & 112 & 0.30 & 0.450 & 0.8 & 2.5 & -1.2 & 139 \\
\hline & 113 & 0.30 & 0.450 & 0.5 & 2.3 & -1.2 & 143 \\
\hline & 114 & 0.30 & 0.450 & 0.9 & 2.0 & -1.0 & 143 \\
\hline \multirow[t]{9}{*}{$82 \mathrm{~Pb}$} & 108 & 0.35 & 0.375 & -0.4 & 2.4 & - & 124 \\
\hline & 109 & 0.30 & 0.450 & -0.5 & 2.4 & - & 132 \\
\hline & 110 & 0.30 & 0.450 & -0.7 & 2.7 & -2.5 & 136 \\
\hline & 111 & 0.30 & 0.450 & -0.1 & 2.6 & -2.1 & 139 \\
\hline & 112 & 0.30 & 0.450 & 0.3 & 2.9 & -1.8 & 143 \\
\hline & 113 & 0.30 & 0.450 & -0.2 & 2.7 & -1.9 & 147 \\
\hline & 114 & 0.35 & 0.450 & 0.1 & 2.1 & -1.5 & 154 \\
\hline & 115 & 0.35 & 0.375 & 0.7 & 2.1 & -1.5 & 147 \\
\hline & 116 & 0.35 & 0.375 & 1.1 & 2.0 & -1.3 & 142 \\
\hline
\end{tabular}




.

$\dot{0}$


IFIGURE CAPTIONS

Fig. 1 Contours of well depth for the banana minimum at $I=30$. The letters represent the deformation parameters. For a given value of 2 , all isotopes not characterized by a deformation have the same deformation as those with one less neutron. The symbols are: $A=\left(\nu_{2}=0.25 ; \nu_{31}=0.375\right)$; $B=\left(\nu_{2}=0.30 ; \nu_{31}=0.375\right) ; C=\left(\nu_{2}=0.35, \nu_{31}=0.375\right) ; D=\left(\nu_{2}=0.40, \nu_{31}=0.375\right) ;$ $E=\left(\nu_{2}=0.30, \nu_{31}=0.450\right) ; F=\left(\nu_{2}=0.35, \nu_{31}=0.450\right) ; G=\left(\nu_{2}=0.35, \nu_{31}=0.525\right) ;$ and $\mathrm{H}=\left(\nu_{2}=0.25 ; \nu_{31}=0.450\right)$

Fig. 2 Contours of the excitation energy of the banana minimum at $I=40$. The numbers give representative values of the static moments of inertia.

Fig. 3 Neutron Single-Particle Energy as a function of rotational frequency <Jy> for deformation parameters $\nu_{2}=0.30, \nu_{4}=-0.02$ and $\nu_{31}=0.450$.

Fig. 4 Proton Single-Particle Energy as a function of $\langle J y\rangle$. Deformation parameters as in $\mathrm{fig} \cdot(3)$.

Eig. 5 Neutron Alignments $\langle J y\rangle$; orbitals are characterized by their neutron number. Deformation parameters as in fig. (3).

Fig. 6 Proton Alignments <Jy>; orbitals are characterized by their proton number. Deformation Parameters as in fig. (3). 
ANL-P.20,4:3

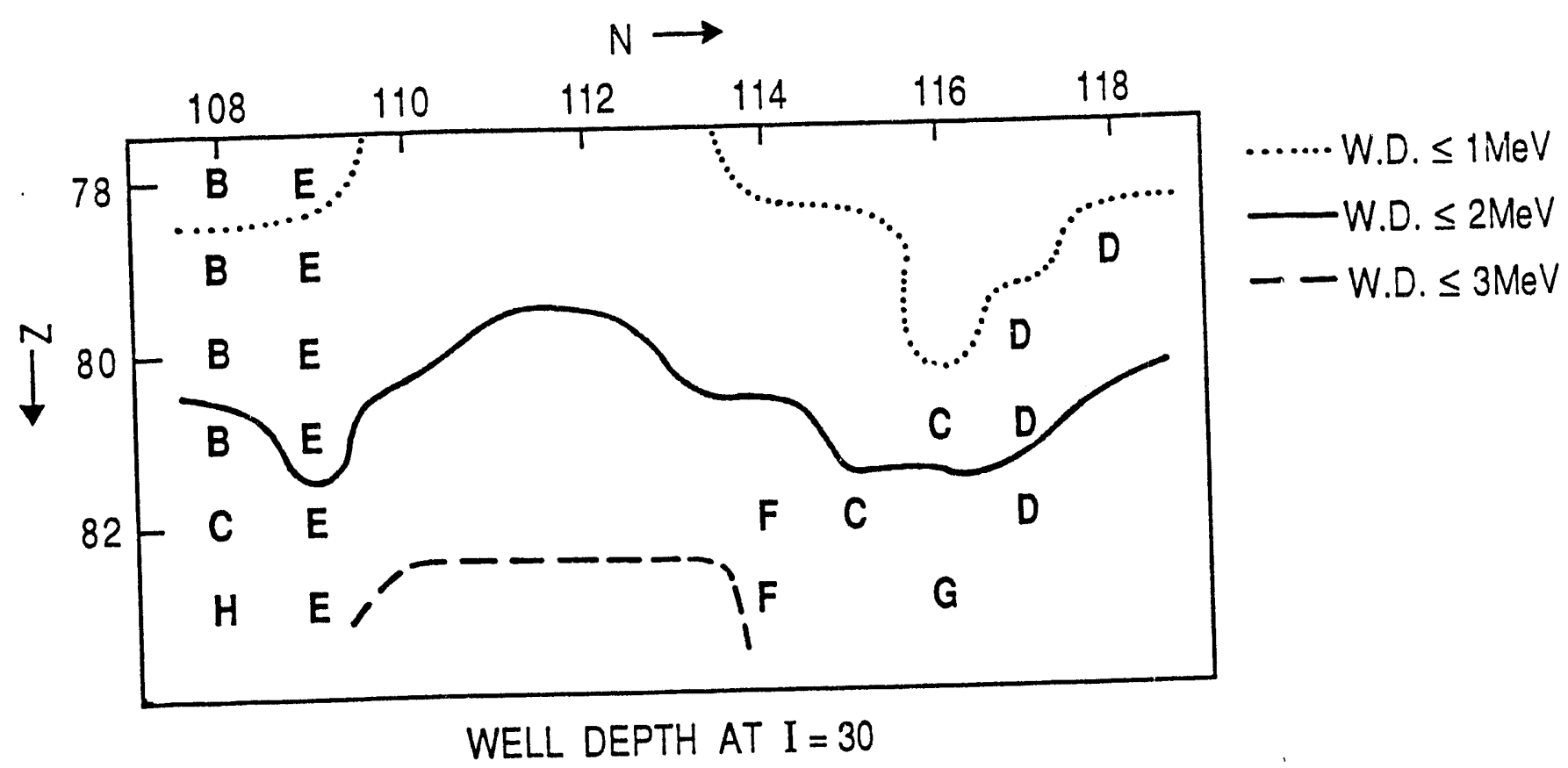

Fig. 1 
ANL-P. 20,418

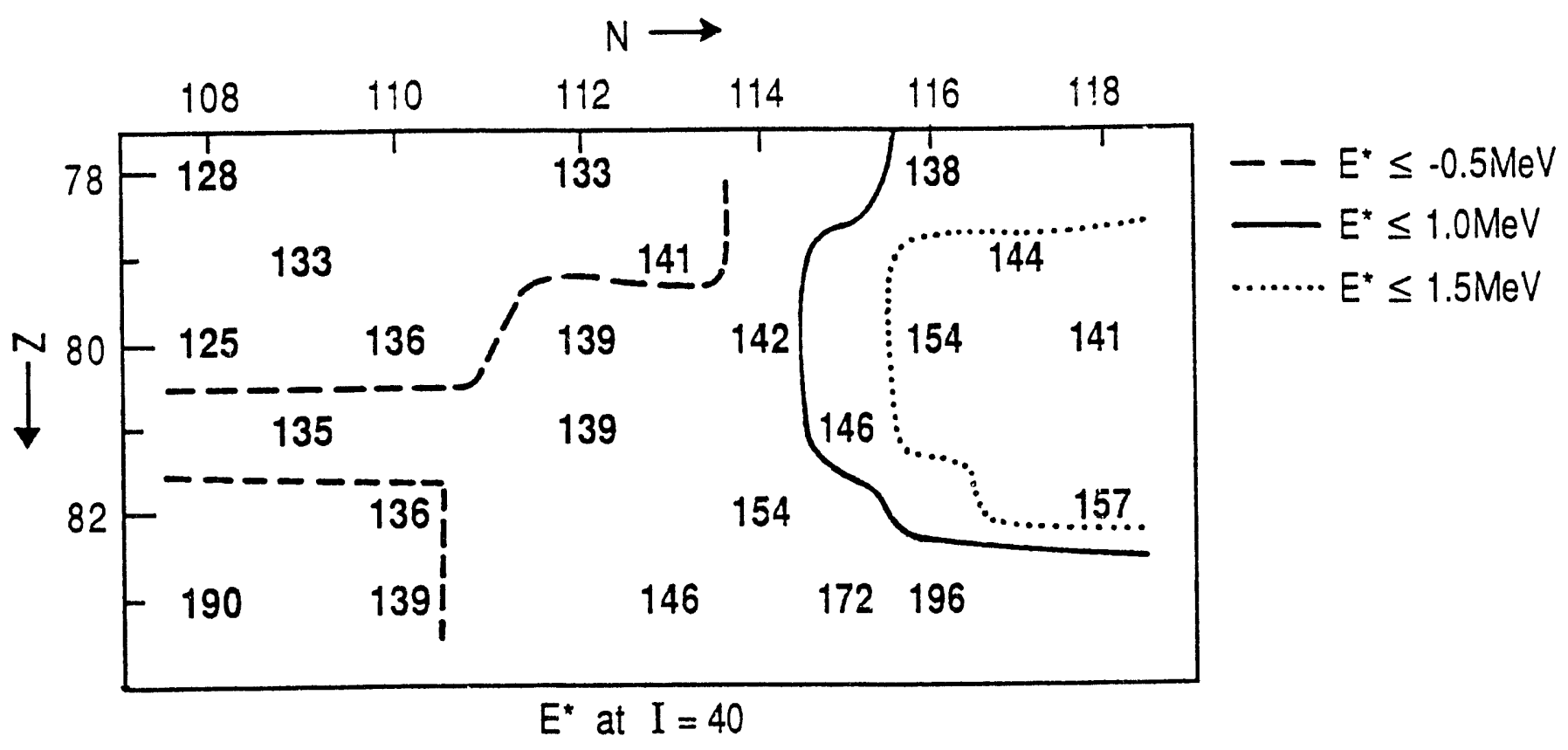

Fig. 2 
ANL-P-20,416

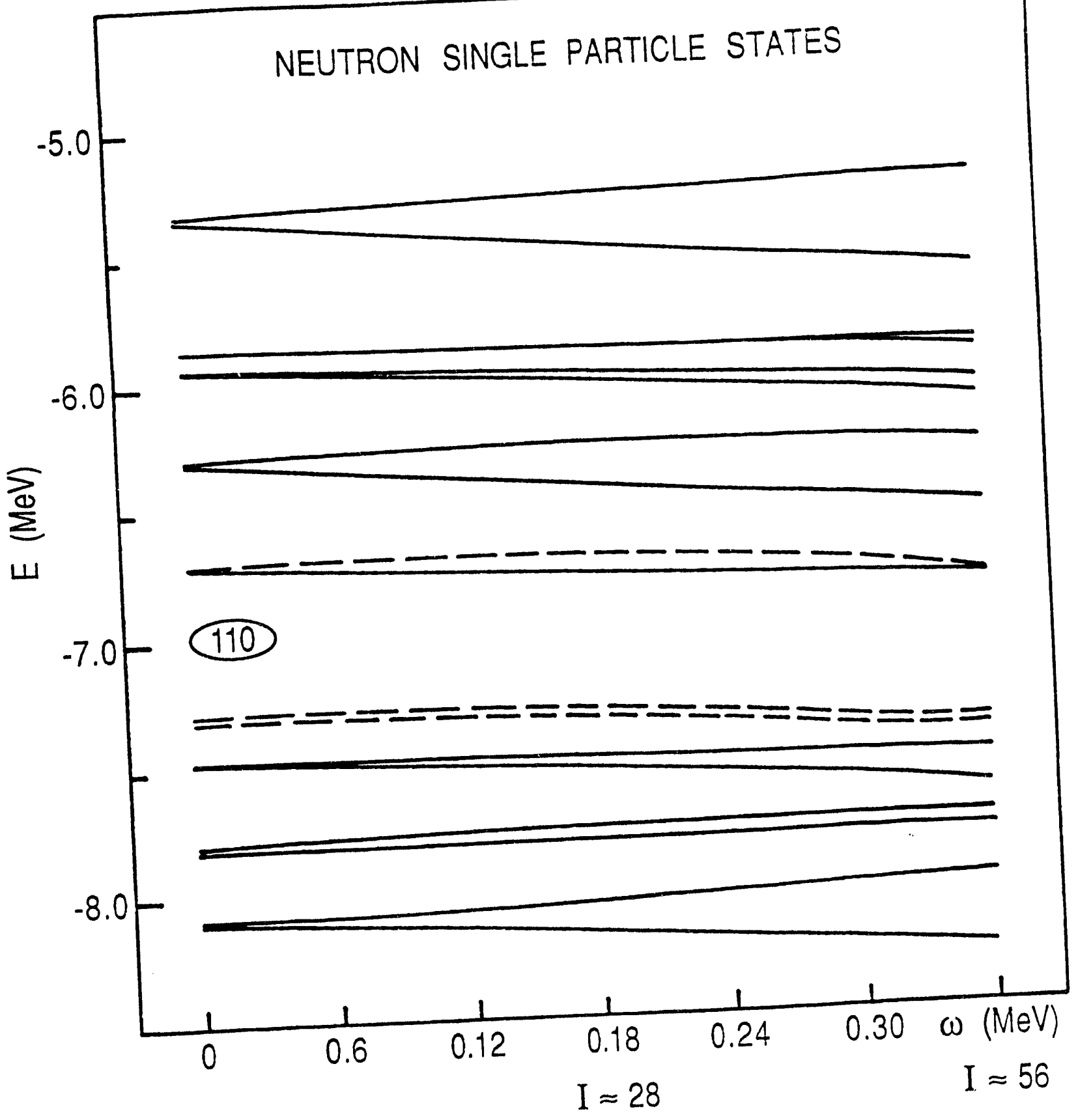

Fig. 3 


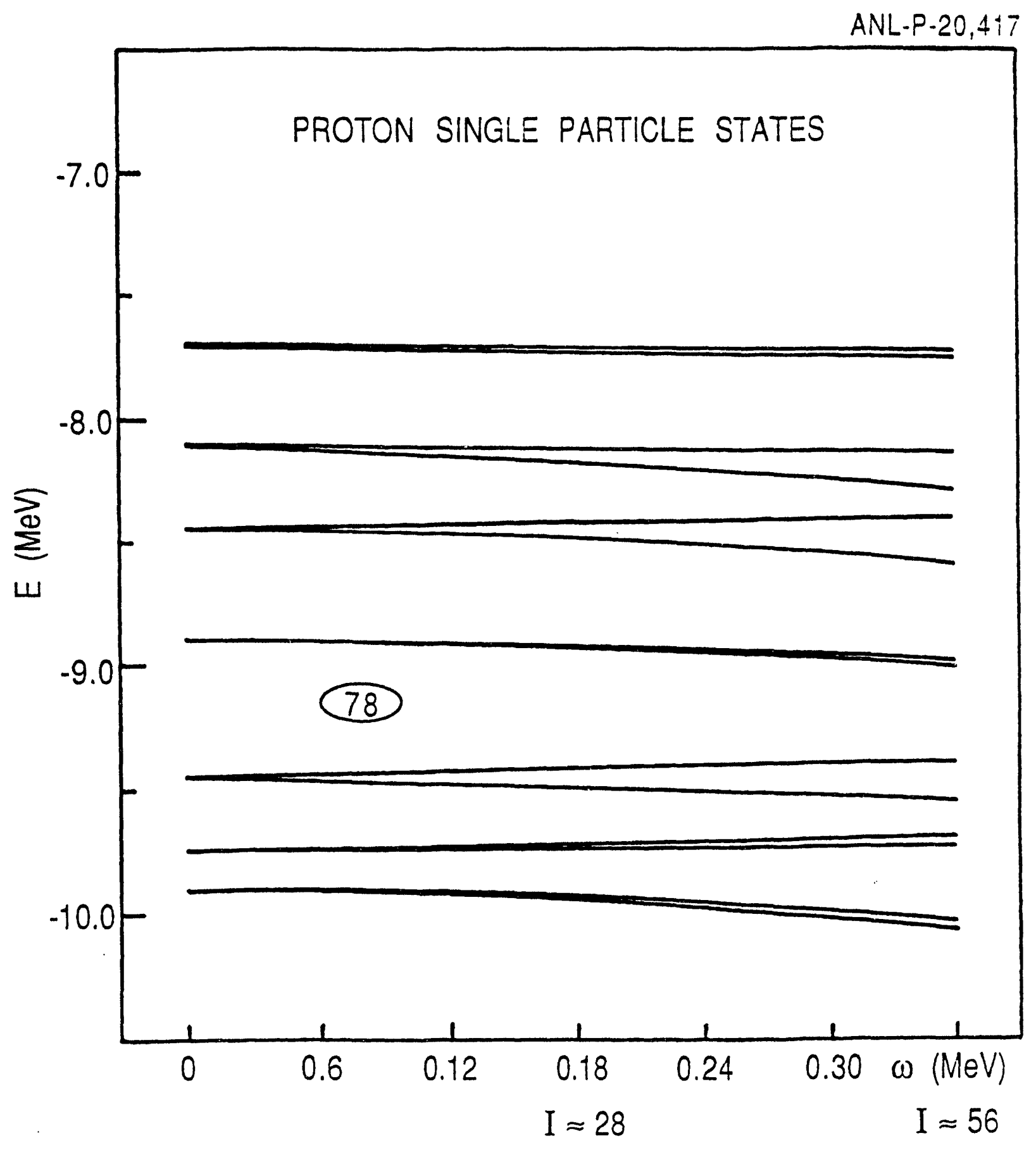

Fig. 4 


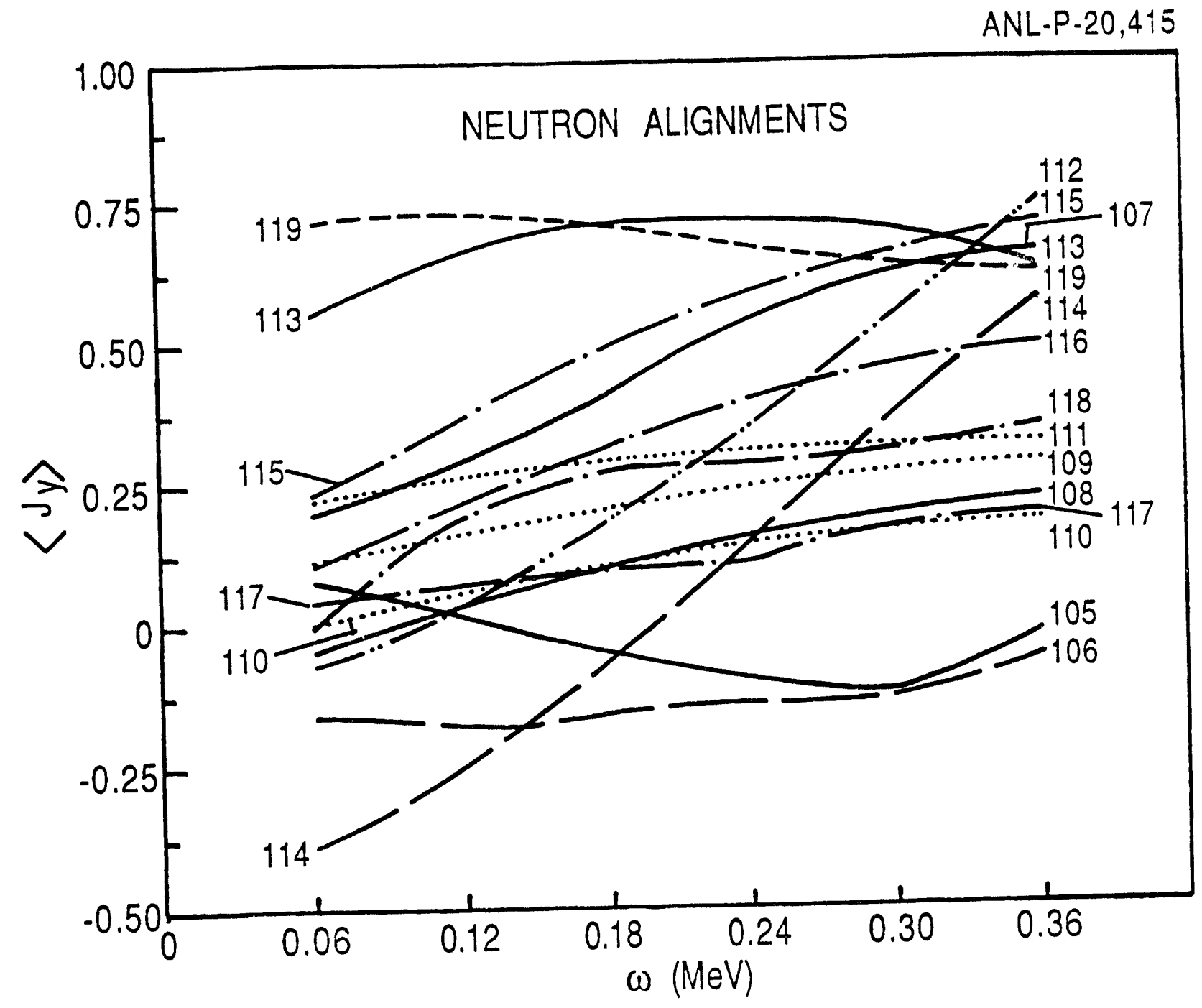

Fig. 5 


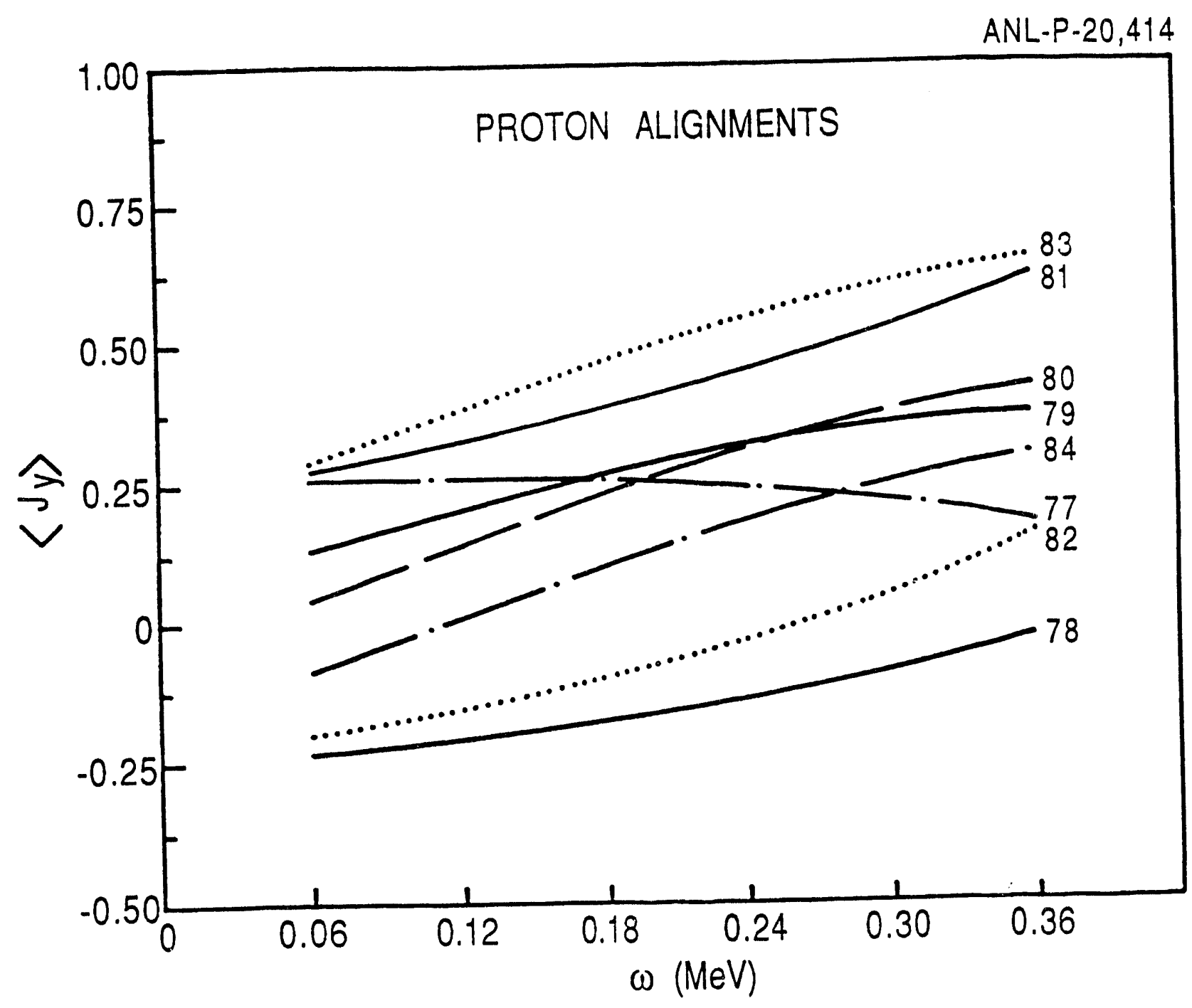

Fig. 6 



$$
\text { n }
$$

$\mid$ 